\title{
Development of nanofluorapatite polymer-based composite for bioactive orthopedic implants and prostheses
}

This article was published in the following Dove Press journal:

International Journal of Nanomedicine

II August 2014

Number of times this article has been viewed

\author{
Gangfeng $\mathrm{Hu}^{1,2}$ \\ Hui Wang' \\ Xiaocong Yao' \\ Dawei $\mathrm{Bi}^{\prime}$ \\ Gang Zhu' \\ Songchao Tang ${ }^{2}$ \\ Jie $\mathrm{Wei}^{2}$ \\ Lili Yang ${ }^{3}$ \\ Peijian Tong ${ }^{4}$ \\ Luwei $\mathrm{Xiao}^{4}$ \\ 'The First People's Hospital of \\ Xiaoshang, Hangzhou, People's \\ Republic of China; ${ }^{2}$ Key Laboratory \\ of Ultra-fine Materials, Ministry of \\ Education, East China University \\ of Science and Technology, \\ Shanghai, People's Republic \\ of China; ${ }^{3}$ Department of Orthopedic \\ Surgery, Changzheng Hospital, The \\ Second Military Medical University, \\ Shanghai, People's Republic of China; \\ ${ }^{4}$ Zhejiang Traditional Chinese Medical \\ University, Hangzhou, People's \\ Republic of China
}

Correspondence: Xiaocong Yao The First People's Hospital of Xiaoshang, Shixinnan Road 199, Hangzhou 3 I I200, People's Republic of China

Tel +86 57I 83807999

Email hugfIIII@I63.com

Lili Yang

Department of Orthopaedic Surgery, Changzheng Hospital, The Second Military Medical University, Fengyang Road 4I5,

Shanghai 20003, People's Republic of

China

Tel +86 21 81886999

Email biomaterbone@163.com
Abstract: Fluorapatite with low solubility is a promising biomaterial due to its structure, which is similar to hydroxyapatite. In this study a bioactive composite of nanofluorapatite (n-FA) and polyamide 12 (PA12) was fabricated. The results revealed that the mechanical properties (such as compressive strength and elastic modulus), hydrophilicity, and antibacterial properties of n-FA/PA12 composite were obviously improved by adding n-FA into PA12 as compared with PA12. In addition, cell proliferation of MC3T3-E1 cells cultured on n-FA/PA12 composite was significantly higher than with PA12, and alkaline phosphatase activity of MC3T3-E1 cells on the n-FA/PA12 composite was expressed at obviously higher levels as compared with PA12. The results suggest that $\mathrm{n}-\mathrm{FA} / \mathrm{PA} 12$ composite could support cell proliferation and differentiation, showing good cytocompatibility. Histological evaluation indicates that n-FA/PA12 composite enhances the efficiency of new bone formation with the introduction of n-FA into PA12, and the quantity of the newly formed bone for n-FA/PA12 composite is significantly higher than with PA12. In conclusion, n-FA/PA12 composite exhibits good biocompatibility and osteogenesis, which might be used for various orthopedic prostheses and dental implants.

Keywords: nanofluorapatite, bioactive composites, cell behavior, orthopedic implants

\section{Introduction}

Hydroxyapatite (HA) biomedical materials have attracted extensive attention over the past decades because of the similarity in chemical composition and crystallographic structure to those of hard tissues (such as tooth and bone) in humans. ${ }^{1}$ Fluorapatite (FA), in which fluorine takes the place of the hydroxyl group in HA, was recognized as a promising biomedical material for bone and tooth implants because of its structural similarity to HA, and FA has the additional benefit of fluorine release. ${ }^{2}$ In addition, the physiological significance of fluorine ions in promoting mineralization and crystallization of calcium phosphate in bone formation has been extensively studied. ${ }^{3,4}$

Similar to HA, FA itself cannot be used for heavy load-bearing applications for bone substitutes due to its poor mechanical properties. Thus, most studies were focused on the mechanical or biological properties of FA in the form of coating. ${ }^{5}$ The FA coatings were stable and did not exhibit signs of dissolution and degradability, and FA can develop chemical bonds with bone tissue, which is stronger than bone or bioceramics alone. ${ }^{6-8}$ Both in vivo and in vitro studies have demonstrated that FA has a lower bioresorption rate than HA and retained biocompatibility comparable with HA in terms of fixation to bones and bone ingrowth. ${ }^{9}$

Biocomposites containing bioactive inorganic materials and polymers have gained much interest and are perceived to be beneficial over conventional inorganic

submit your manuscript | www.dovepress.con 
materials or polymeric biomaterial alone in the field of bone grafting. ${ }^{10,11}$ Therefore, a lot of biocomposites containing bioactive inorganic materials and polymers have been studied as bone regeneration materials. ${ }^{12,13}$ Furthermore, emphasis has been placed on the development of bioactive composites of nanoinorganic materials and polymers in the biomedical field for good biological and mechanical performances, wherein nanobioactive material particles are made to disperse in polymeric matrices. ${ }^{12-14}$ The addition of nanobioactive ceramic into polymers could not only introduce bioactivity but also improve the mechanical properties of the composites. ${ }^{15,16}$ The low solubility of FA is beneficial to improve osseointegration at implant-bone interface, which is considered a promising biomedical material for bone fixation. ${ }^{17,18}$ Polyamides (PAs) are the preferred polymer material for orthopedics and dentistry due to their high strength and good biocompatibility. ${ }^{19,20}$ Polyamide 12 (PA12) is one of the polymeric materials in the PA family. Until now there has been no information on the fabrication of FA/PA12 bioactive composites. Therefore, in this study nanofluorapatite (n-FA) was added into PA12 to form n-FA/PA bioactive composite, and the mechanical, hydrophilic, and antibacterial properties of both in vitro cells and in vivo bone tissue responses to n-FA/PA12 composites were reported.

\section{Materials and methods Preparation of $n-F A$}

n-FA was synthesized using a chemical precipitation method. Diammonium hydrogen phosphate $\left(\left[\mathrm{NH}_{4}\right]_{2} \mathrm{HPO}_{4}\right)$ and ammonium fluoride $\left(\mathrm{NH}_{4} \mathrm{~F}\right)$ were dissolved together in aqueous solution. Aqueous solution of calcium nitrate $\left(\mathrm{Ca}\left[\mathrm{NO}_{3}\right]_{2} \cdot 4 \mathrm{H}_{2} \mathrm{O}\right)$ was added dropwise into the mixed solution of $\left(\mathrm{NH}_{4}\right)_{2} \mathrm{HPO}_{4}$ and $\mathrm{NH}_{4} \mathrm{~F}$. The $\mathrm{pH}$ value of the solution was kept at 10 12 by adding ammonium hydroxide. After the precipitation reaction was complete, FA precipitate was obtained and fully washed with deionized water. FA precipitation in a solid-solution ratio of $2 \mathrm{wt} \%$ was treated hydrothermally in an autoclave at $140^{\circ} \mathrm{C}$ under $0.3 \mathrm{MPa}$ for 2-4 hours. After treatment, the FA precipitation became n-FA in a slurry state. The phase composition and morphology of n-FA were characterized using transmission electron microscopy (TEM) (JEM 2010, JEOL, Tokyo, Japan), energy dispersive spectroscopy (EDS) (Falcon, Mahwah, NJ, USA), and X-ray diffraction (XRD) (Geigerflex, Rigaku Corporation, Tokyo, Japan).

\section{Preparation of n-FA/PAI 2 composite}

n-FA and PA12 (purchased from Evonik Degussa Co., Ltd, Shanghai, People's Republic of China) composites with $40 \mathrm{w} \% \mathrm{n}-\mathrm{FA}$ were prepared by the cosolution method in which n-FA slurry was mixed with PA12 and N,Ndimethylacetamide solution. When PA12 was added, the mixture was stirred and the temperature was gradually increased to $140^{\circ} \mathrm{C}$ and kept for 4 hours, then cooled down to room temperature. After being fully washed with deionized water and ethanol, the product was dried at $100^{\circ} \mathrm{C}$ for 48 hours. The dense samples of n-FA/PA12 composite with $40 \mathrm{w} \% \mathrm{n}-\mathrm{FA}$ content were prepared using an injection molding machine. PA12 dense samples were prepared the same way and were used as controls. The morphology and microstructure of n-FA/PA12 composite dense samples were observed using scanning electron microscopy (SEM) (S-3400N; Hitachi Ltd, Tokyo, Japan). The porous scaffolds of n-FA/PA12 composites for animal experiments were made by an injection foaming method with the same machine, and barium azodicarboxylate was used as a foaming agent. Surface morphology of the n-FA/PA12 composite scaffolds was examined by SEM.

\section{Mechanical, hydrophilic, and antibacterial properties}

The mechanical properties of n-FA/PA12 composite (PA12 samples as controls), dense samples of the size $\Phi 6 \times 10 \mathrm{~mm}$ (diameter $6 \mathrm{~mm}$ and height $10 \mathrm{~mm}$ ), were measured using a universal mechanical testing machine (QTESTTM/10; MTS Systems Co., Eden Prairie, MN, USA) according to ASTM International/International Organization for Standardization (ISO) (D695-08 and ISO 604-2002) standards at a crosshead speed of $2 \mathrm{~mm} / \mathrm{min}$, and the load was applied until the samples were compressed to about $30 \%$ in height. PA12 samples were used as controls. The compressive strength and elastic modulus were calculated within the linear range of the stress-strain curve. The surface hydrophilicity of the samples was assessed by measuring the contact angle. The samples were mounted on a stage of a G10 Krüss contact angle goniometer (Krüss $\mathrm{GmbH}$, Hamburg, Germany), and water contact angle was obtained by the sessile drop method at room temperature. A water droplet was placed on the sample surface and contact angle was measured after 10 seconds.

The Escherichia coli strain (ATCC 25922 [American Type Culture Collection, Manassas, VA, USA], provided by the Laboratory of Pathogenic Microorganisms, Basic Medical College of Shanghai Jiao Tong University, Shanghai, People's Republic of China) was used to test the bacterial adherence on n-FA/PA12 composite dense samples $(\Phi 12 \times 2 \mathrm{~mm})$ and PA12 samples as controls. The $E$. coli strain 
was cultured at $37^{\circ} \mathrm{C}$ overnight in Trypto Soy Broth. The mixture was diluted at a ratio of 1:1,000 in Trypto Soy Broth with $0.25 \%$ glucose, and $1 \mathrm{~mL}$ of the bacterial suspension ( $10^{5}$ colony forming units) was inoculated into 24 -well tissue culture plates and the samples placed in the wells. After being cultured for 24 hours, the bacteria without adherence were removed and the composite samples were rinsed twice with $2 \mathrm{~mL}$ phosphate buffered saline (PBS). The samples were transferred into new tubes with $10 \mathrm{~mL}$ PBS after rinsing and then were ultrasonically washed with water for 5 minutes to remove adherent bacteria. The number of viable bacteria in the solution was counted using the cultural method and Pearlcore Staphylococcus Medium.

\section{Cell proliferation and morphology}

Prior to cell seeding, the experimental samples were sterilized in an autoclave at $120^{\circ} \mathrm{C}$ for 30 minutes. Preosteoblasts, MC3T3-E1 cells, were purchased from Cambrex Bio Science Walkersville, Inc. (Walkersville, MD, USA). Culture medium $(1 \mathrm{~mL})$ with a density of $4 \times 10^{5}$ cells $/ \mathrm{mL}$ was seeded on top of each n-FA/PA12 composite dense sample $(\Phi 12 \times 2 \mathrm{~mm})$, and PA12 samples as controls, followed by incubation for 1 day, 3 days, and 5 days. Composite-cell constructs were then placed in culture medium containing 3-(4,5dimethylthiazol-2-yl)-2,5-diphenyltetrazolium bromide (MTT) and incubated in a humidified atmosphere at $37^{\circ} \mathrm{C}$ for 4 hours. Cell proliferation was determined by MTT assay (MTT Kit, Roche Diagnostics Corporation, IN, USA). The absorbance value was measured at $595 \mathrm{~nm}$ with a microplate reader. Results are reported as optimal density (OD) units. The morphologies of the MC3T3-E1 cells cultured on both the n-FA/PA12 composite and PA12 were observed using an inverted light microscope (Olympus IMT-2, A10PL; Olympus Corporation, Tokyo, Japan).

MC3T3-E1 cells were cultured in $\alpha$-Modified Eagle's Medium (Life Technologies, Carlsbad, CA, USA) containing 10\% fetal calf serum (Life Technologies), $100 \mu \mathrm{g} / \mathrm{mL}$ streptomycin (Amresco LLC, Cleveland, OH, USA) and $100 \mu \mathrm{g} / \mathrm{mL}$ penicillin (Amresco LLC) at $37^{\circ} \mathrm{C}$ in a humidified atmosphere of $5 \%$ (volume/volume $[\mathrm{v} / \mathrm{v}]$ ) carbon dioxide. The cell culture medium was changed every 3 days. SEM was used for investigation of cell morphology on the scaffold. The cell-loaded scaffolds were rinsed with PBS after 3 days of cell seeding and fixed in glutaraldehyde $2.5 \%$ for 1 hour. For dehydrating, the samples were placed in sequentially increasing ethanol concentration to $100 \%$. After drying, the samples were coated with gold using a sputter coater and prepared for SEM analysis.

\section{Alkaline phosphatase activity}

MC3T3-E1 cells with a density of $4 \times 10^{5}$ were seeded on the n-FA/PA12 composites ( $\Phi 12 \times 2 \mathrm{~mm})$, and PA12 samples as controls, and alkaline phosphatase (ALP) activity was tested at 4 days and 7 days. The adherent cells were removed from samples and were washed with PBS, followed by adding a cell lysis buffer containing $0.1 \%$ Triton X-100 to the samples and freezing to $-20^{\circ} \mathrm{C}$. The frozen samples were thawed at $37^{\circ} \mathrm{C}$ for 5 minutes in order to test the ALP activity, following the manufacturer's instructions (ALP kit 104, Sigma-Aldrich Co., St Louis, MO, USA). The number of cells was determined by measuring deoxyribonucleic acid (DNA) content using Quant-iT ${ }^{\text {TM }}$ PicoGreen dsDNA Reagent and Kits (Molecular Probes, Eugene, OR, USA). The DNA of the samples was quantitated by measuring the fluorescence using Synergy HT Multi-Detection Microplate Reader (BioTek, Winooski, VT, USA) at wavelengths of $480 \mathrm{~nm}$ excitation and $520 \mathrm{~nm}$ emission, respectively. Enzyme activity was quantified by absorbance measurements at $405 \mathrm{~nm}$, and ALP activity was calculated from a standard curve after normalizing to the total DNA content. The results were expressed in nanomoles of p-nitrophenol produced per minute per nanogram of DNA.

\section{Implantation in vivo}

Biocompatibility and osteogenesis in vivo of the n-FA/ PA12 composite were evaluated using histological methods, and PA12 samples as controls. Twelve healthy New Zealand white rabbits with an average weight of $3 \mathrm{~kg}$ were used for the implantation. Under general anesthesia and sterile conditions the left femur of each rabbit was exposed and one defect $(\Phi 6 \mathrm{~mm})$ was drilled in the distal part of the femur. The bone cavities were washed to eliminate bone debris and dried with gauze. Porous samples of n-FA/PA12 composite of the size $\Phi 6 \times 6 \mathrm{~mm}$ were implanted into the defects in the rabbit femora. Three animals of each group were sacrificed by an abdominal injection overdose of pentobarbital sodium at 4 weeks and 8 weeks after implantation.

The samples, together with surrounding tissue, were excised, fixed in 10\% neutral buffered formalin, decalcified, and embedded in paraffin. Tissue blocks were sectioned to $4 \mu \mathrm{m}$ in thickness, stained with hematoxylin and eosin (H\&E), and observed with a light microscope (CX21, Olympus Corporation). To quantitatively determine the amount of newly formed bone, statistical analysis of the histological sections was performed at different implantation times (4 weeks and 8 weeks). Three pieces of histological sections were randomly selected from each group. After H\&E staining, each section was observed under light microscope, and at 
least five images were randomly obtained in the same section. Using image analytical software Image-ProPlus (Media Cybernetics, Inc., Rockville, MD, USA), new bone area was expressed as percentage of newly formed bone area within the original drill defect area.

\section{Statistical analysis}

Statistical analysis was performed using one-way analysis of variance with post hoc tests. All the data are expressed as mean \pm standard deviation. Differences were considered statistically significant at $P<0.05$.

\section{Results \\ Morphology and composition of $n$-FA}

Figure 1A presents the TEM image of n-FA. It can be seen that the morphology of the synthesized n-FA was uniformly rod-like grains with a size of around $40 \mathrm{~nm}$ in diameter and $100 \mathrm{~nm}$ in length. The EDS pattern of n-FA is shown in Figure 1B. It can be seen that fluoride $(F)$ presented in the n-FA. The molar ratio of calcium to phosphorus for both n-FA and n-HA was almost the same (1.67) according to the results from EDS. The XRD pattern of n-FA is shown in Figure 1C. It was found that the prepared sample of n-FA consisted of an apatite phase, according to the standard pattern of FA (Joint Committee on Powder Diffraction and Standards [JCPDS] 15-0876). The n-FA is different from hydroxyapatite because of the replacement for the $\mathrm{OH}^{-}$group with $\mathrm{F}^{-}$in the HA lattice. ${ }^{2,5} \mathrm{FA}$ is more stable and has lower degradation in vivo in comparison with $\mathrm{HA}$, due to the smaller size of $\mathrm{F}^{-}$in comparison with the $\mathrm{OH}^{-}$group..$^{2,5}$

\section{Mechanical and surface hydrophilic properties of composite}

Table 1 presents the mechanical and surface hydrophilic properties of $n-F A / P A 12$ composites. It was found that the n-FA/PA12 composite with $40 \mathrm{w} \%$ n-FA content resulted in the higher compressive strength $(105 \pm 4 \mathrm{MPa})$

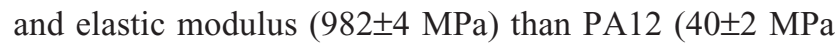
and $461 \pm 4 \mathrm{MPa})$. The results revealed that the mechanical properties of n-FA/PA12 composite were significantly higher than those of PA12 $(P<0.05)$, indicating that the
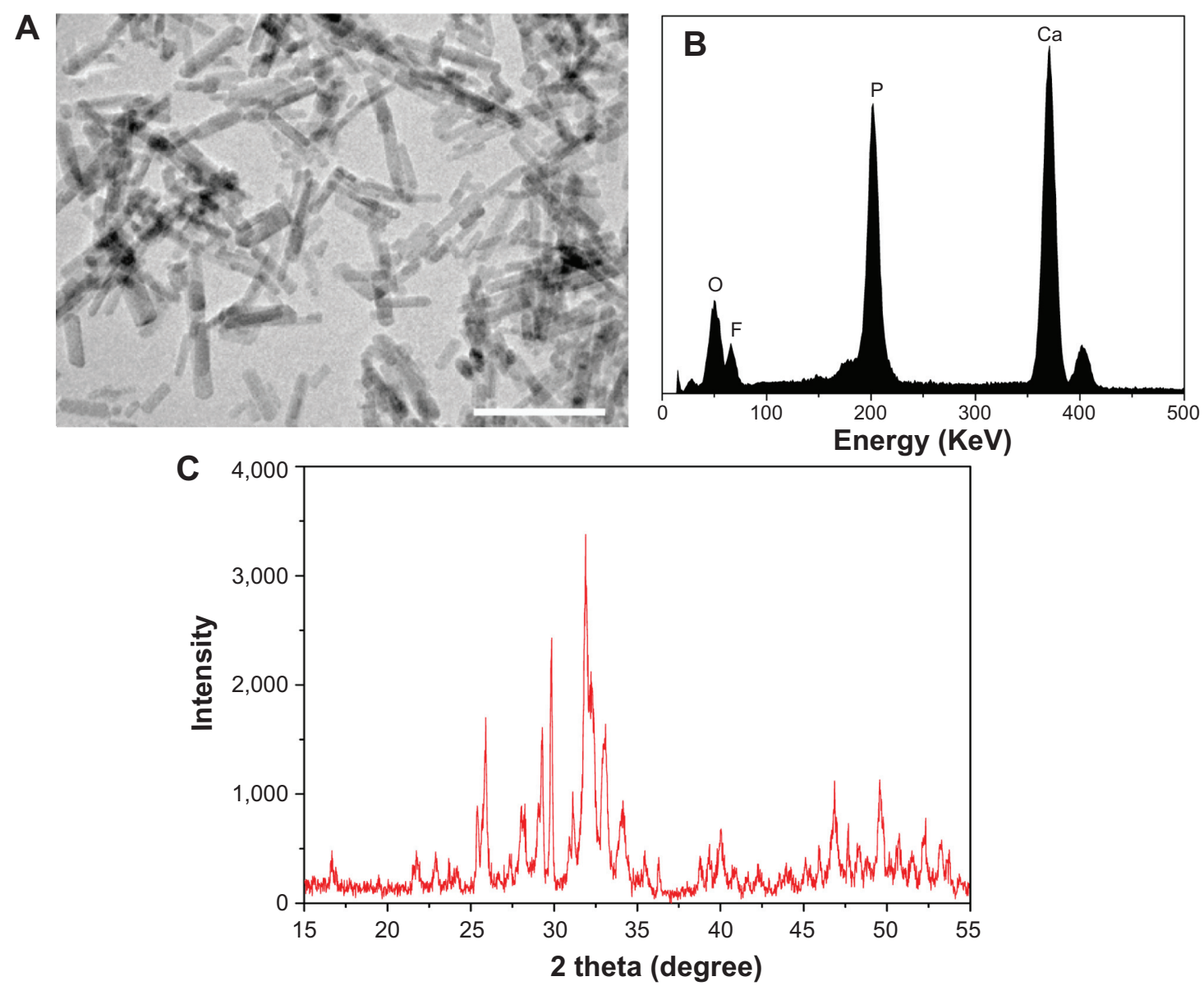

Figure I Transmission electron microscopy image (A), energy dispersive spectroscopy (B), and X-ray diffraction (C) patterns of nanofluorapatite (bar represents I00 nm). 
Table I Mechanical properties and water contact angles of nanofluorapatite (n-FA)/polyamide 12 (PAI2) composite with 40 wt $\%$ n-FA and PAI 2

\begin{tabular}{llll}
\hline Samples & $\begin{array}{l}\text { Compressive } \\
\text { strength }(\mathbf{M P a})\end{array}$ & $\begin{array}{l}\text { Elastic modulus } \\
(\mathbf{M P a})\end{array}$ & $\begin{array}{l}\text { Water contact } \\
\text { angles }\left(^{\circ}\right)\end{array}$ \\
\hline $\begin{array}{l}\text { 40 n-FA/PAI2 } \\
\text { composite }\end{array}$ & $105 \pm 4$ & $982 \pm 4$ & $2 \mathrm{I} \pm 2$ \\
PAI2 & $40 \pm 2$ & $46 \mathrm{I} \pm 4$ & $58 \pm 3$ \\
\hline
\end{tabular}

addition of n-FA into PA12 improved the mechanical properties of composites. The hydrophilic properties of the composite surfaces were evaluated by measuring the water contact angles. The water contact angles of PA12 and n-FA/PA12 composite with $40 \mathrm{w} \% \mathrm{n}$-FA content were $58^{\circ} \pm 3^{\circ}$ and $21^{\circ} \pm 2^{\circ}$, respectively. The water contact angle of n-FA/PA12 composite was significantly lower than that of PA12 $(P<0.05)$, indicating that the addition n-FA into PA12 could enhance the hydrophilicity of the n-FA/ PA12 composites.

\section{Surface morphology and antibacterial property of composite}

Figure 2 shows SEM images of the surface morphology and microstructure of n-FA/PA12 composite and PA12. It was found that n-FA particles were homogeneously distributed into the PA12 matrix and closely combined with PA12 in the composite. The results indicate that the cosolution method used in the study is a very useful way to fabricate inorganic/organic polymer-based composite with good homogeneity. Figure 3 shows the number of viable bacteria (E. coli) adherence on both the n-FA/ PA12 composite and PA12 surfaces. It was found that the number of viable bacteria on n-FA/PA12 composite was lower than that on PA12 $(P<0.05)$, indicating inhibition of bacteria attachment and good antibacterial property of n-FA/PA12 composite.

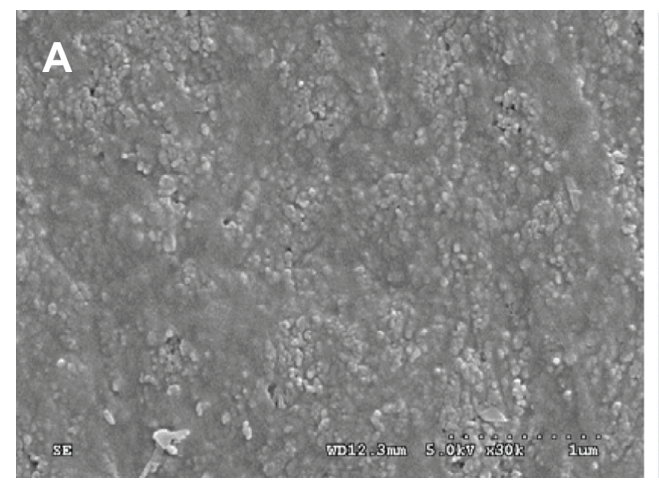

\section{Cell proliferation and morphology}

Figure 4 presents the proliferation of MC3T3-E1 cells cultured on both n-FA/PA12 composite and PA12 assessed using MTT assay. The OD values of both n-FA/PA12 composite and PA12 increased with time, and the OD values of n-FA/ PA12 composite were significantly higher than for PA12 at 3 days and 5 days (no significant difference at 1 day). The results suggest that the n-FA/PA12 composite facilitated cell growth and could promote cell proliferation.

Figure 5 shows the morphology of MC3T3-E1 cells cultured on both n-FA/PA12 composite and PA12. The population of the MC3T3-E1 cells on both samples increased with time, and the proliferation of the cells was normal, indicating good cytocompatibility. In addition, the population of the MC3T3-E1 cells for n-FA/PA12 composite increased faster than for PA12, indicating that the n-FA/PA12 composite could promote cell proliferation.

\section{ALP activity}

ALP of MC3T3-E1 cells cultured on both n-FA/PA12 composite and PA12 is shown in Figure 6. At 4 days, ALP was expressed at lower levels, and no significant difference was detected between n-FA/PA12 composite and PA12. ALP activity increased with time and presented higher levels for both n-FA/PA12 composite and PA12 at 7 days. Moreover, ALP activity on the n-FA/PA12 composite was significantly higher than for PA12 at 7 days, indicating that the n-FA/ PA12 composite could promote cell differentiation.

\section{Surface morphology and cell ingrowth into the composite scaffolds}

Figure 7 shows the surface morphology and microstructure of the n-FA/PA12 composite and PA12 porous scaffolds. Two kinds of scaffolds both exhibited a macroporous structure with completely open interconnected pores, and the pores appeared almost spherical in shape, with diameters of

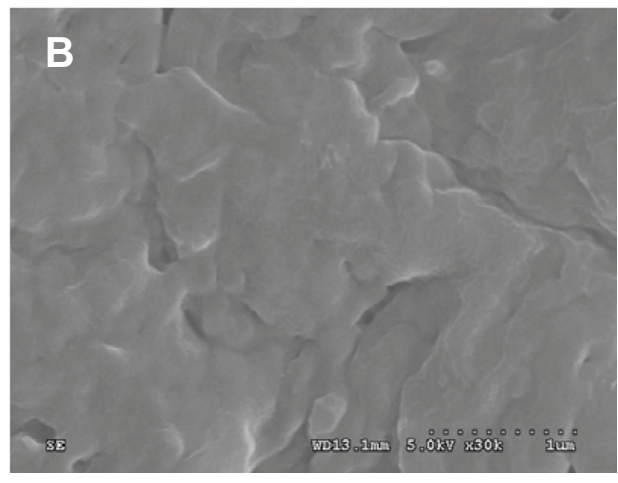

Figure 2 Scanning electron microscopy images of surface morphology of (A) nanofluorapatite (n-FA)/polyamide I2 (PAI2) composite with 40 wt\% n-FA and (B) PAI2. 


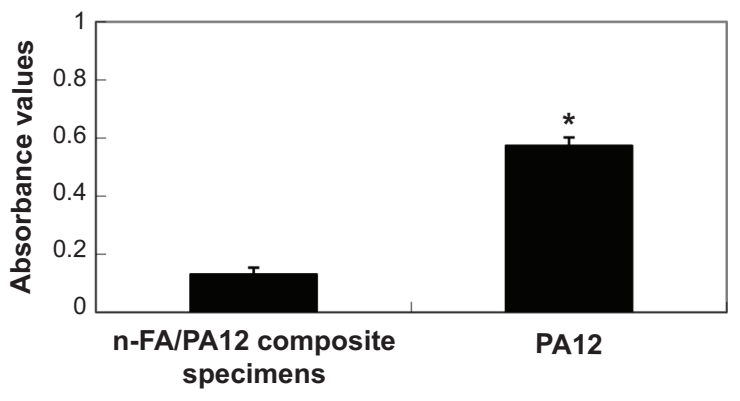

Figure 3 Number of viable bacteria (Escherichia coli) adherence on nanofluorapatite (n-FA)/polyamide 12 (PAI2) composite with 40 wt\% n-FA and PAI2 after 24 hours ( $10^{5}$ colony forming units).

Note: *Indicates significant difference.

400-500 $\mu \mathrm{m}$. Figure 8 presents the SEM images of the morphological features of MC3T3-E1 cells cultured on the n-FA/ PA12 composite and PA12 at 3 days. The MC3T3-E1 cells extended and spread well, exhibiting intimate attachment with the composite scaffold surfaces. The cells formed confluent layers across the pores of the scaffolds. Furthermore, some of the cells attached to the rims of macropores and spread across smaller pores. However, only a few MC3T3-E1 cells spread and attached to the composite scaffold surfaces.

\section{Histological evaluation}

Figure 9 presents the histological evaluation results of n-FA/ PA12 composite scaffold implanted into the defects of the rabbit femora for 4 weeks and 8 weeks. At 4 weeks a few newly formed bone tissues were found to be deposited at the interface between the porous materials and host bone tissue for both n-FA/PA12 composite and PA12, as shown in Figure 9A and 9B. At 8 weeks more newly formed bone tissues were found to have grown into the pores of both n-FA/ PA12 composite and PA12, as shown in Figure 9C and 9D. Quantitative determination of newly formed bone was carried using statistical analysis of histological sections (shown in Figure 10). As for n-FA/PA12 composite, 28\% $03 \%$ and

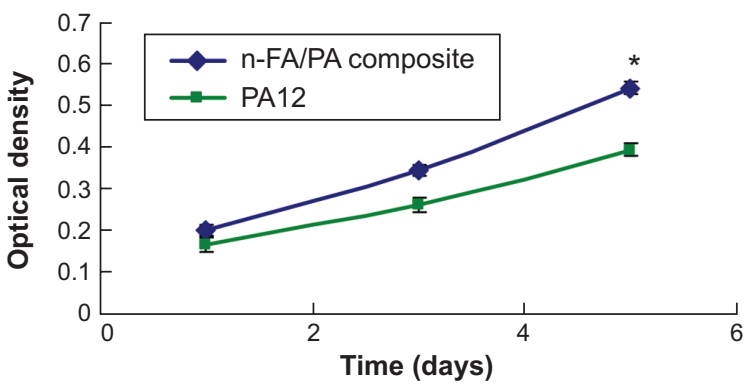

Figure 4 Proliferation of MC3T3-EI cells cultured on nanofluorapatite (n-FA)/ polyamide 12 (PAI2) composite with $40 \mathrm{wt} \% \mathrm{n}$-FA and PAI2 for I day, 3 days, and 5 days.

Note: *Indicates significant difference.
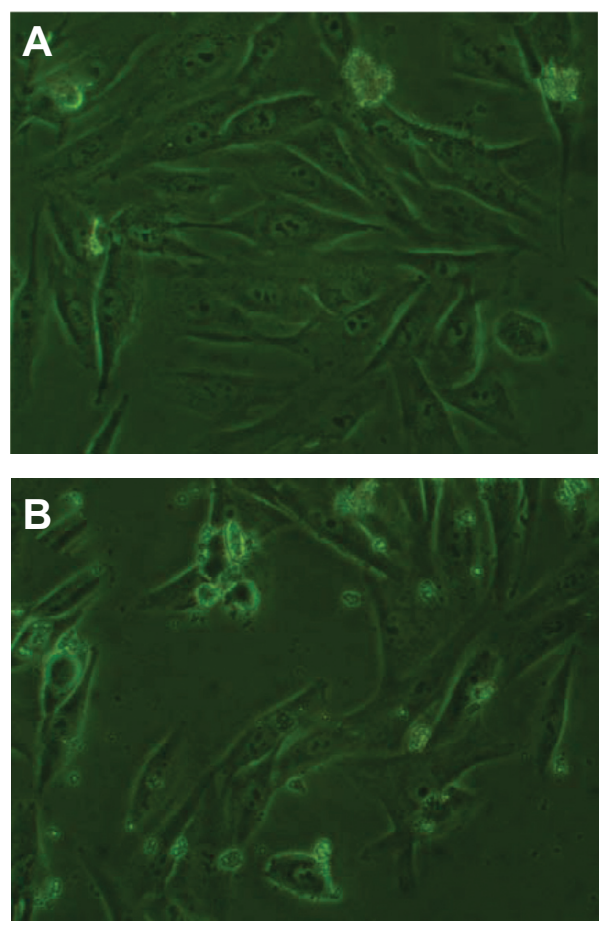

Figure 5 Phase contrast microscopy photographs of MC3T3-EI cells cultured with (A) nanofluorapatite ( $\mathrm{n}$-FA)/polyamide 12 (PAI2) composite with $40 \mathrm{wt} \% \mathrm{n}$-FA and (B) PAI 2 at 3 days.

$62 \% \pm 5 \%$ of the bone defect area was filled with newly formed bone at 4 weeks and 8 weeks, respectively. However, for PA12 the area of newly formed bone was $21 \% \pm 3 \%$ and $53 \% \pm 4 \%$ at 4 weeks and 8 weeks, respectively. The result showed that newly formed bone increased dramatically for n-FA/PA12 composite and PA12 at 8 weeks compared with 4 weeks, and the new bone area for n-FA/PA12 was significantly higher than PA12 composite at both 4 weeks and 8 weeks.

\section{Discussion}

Over the past decades FA as a bioactive material for hard tissue (such as bone and tooth) repair has aroused increasing

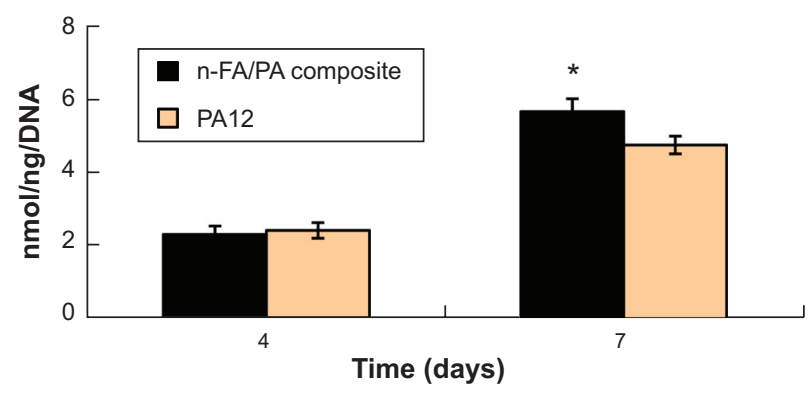

Figure 6 Alkaline phosphatase activity of MC3T3-EI cultured on both nanofluorapatite (n-FA)/polyamide 12 (PAI2) composite with 40 wt\% n-FA and PAI2 at 4 days and 7 days.

Note: *Indicates significant difference. 

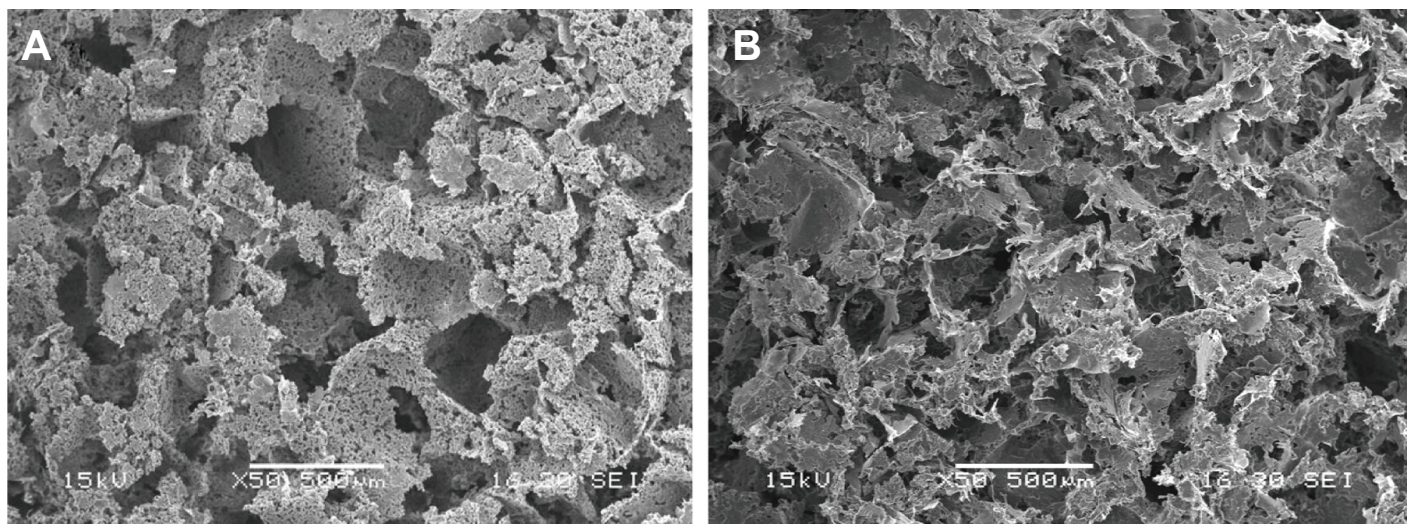

Figure 7 Scanning electron microscopy images of surface morphology of (A) nanofluorapatite (n-FA)/polyamide I2 (PAI2) composite with 40 wt\% n-FA and (B) PAI2.

interest because of its excellent biocompatibility and lower solubility, which could promote osseointegration between the implants and bone tissues. ${ }^{21,22} \mathrm{FA}$ has been used as a coating on titanium implants using the plasma spraying technique, and the results revealed that the osseointegration process of titanium implants coated with FA was superior as compared with uncoated implants. ${ }^{23}$ In this study, n-FA/PA12 bioactive composite was prepared by incorporating n-FA into PA12, and the results revealed that the compressive strength and elastic modulus of n-FA/PA12 composites significantly increased as compared with PA12, indicating that the addition of n-FA into PA12 improved the mechanical properties of composites. In addition, the results showed that incorporating n-FA into PA1 2 markedly improved the hydrophilicity of the n-FA/PA12 composites as compared with PA12. It can be suggested that incorporating hydrophilic inorganic materials into hydrophobic polymers is a viable way to improve polymer hydrophilicity, and more hydrophilic surfaces are better for cell attachment, spreading, and proliferation than hydrophobic surfaces.

Biomaterial-associated infections remain a serious complication in orthopedic surgery, and it has been suggested that incorporation of antibacterial agents into implants can prevent bacterial infections. ${ }^{24}$ Not only could fluoride improve the acid resistance of apatite effectively but also a certain concentration of fluoride could inhibit metabolism of bacteria. ${ }^{25}$ In this study the bacterial adhesion on n-FA/ PA12 composite was determined. The results showed that the n-FA/PA12 composite greatly reduced E. coli adherence as compared with PA12, showing good antibacterial ability. The n-FA/PA12 composite presumably depended on the presence of n-FA or the slow release of fluoride ions from n-FA, which could inhibit $E$. coli attachment. It can be suggested that addition of n-FA into PA12 is an effective way to improve antibacterial properties of n-FA/PA12 composite, which could meet the needs of antibacterial implanted materials.
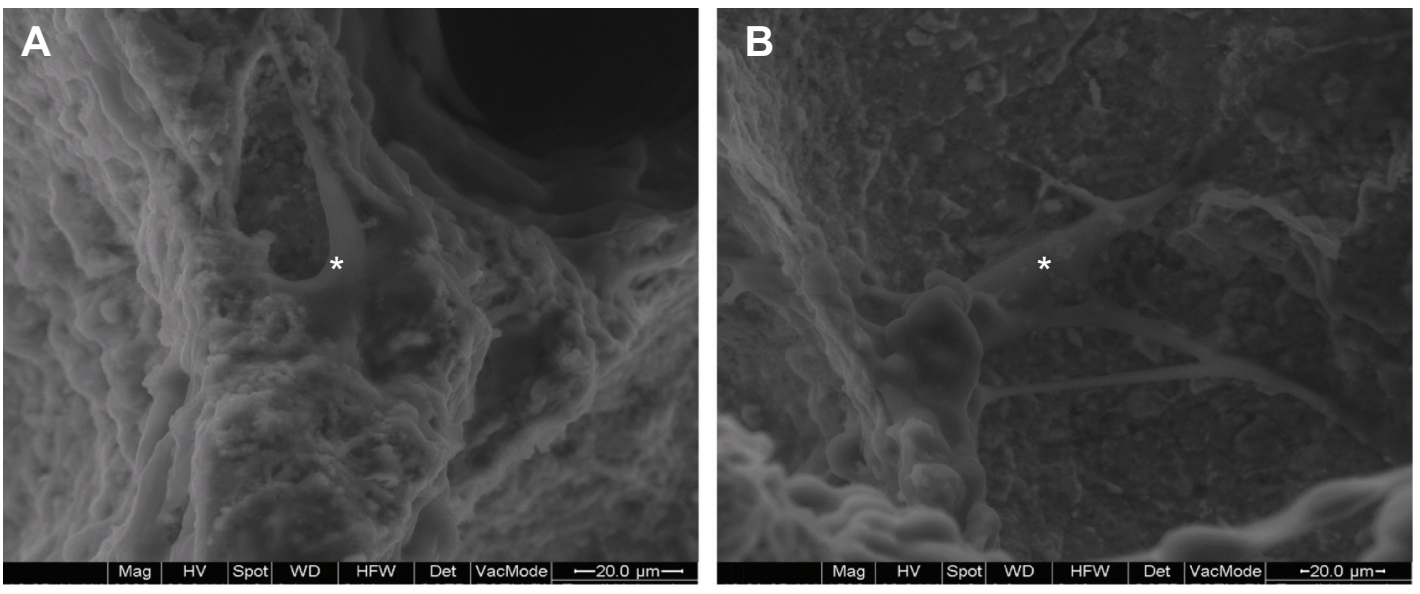

Figure 8 Scanning electron microscopy images of MC3T3-EI cells attached and spread on the surfaces of (A) nanofluorapatite (n-FA)/polyamide I2 (PAI2) composite with $40 w t \% n-F A$ and (B) PAI 2 at 3 days. Note: *Represents cells. 

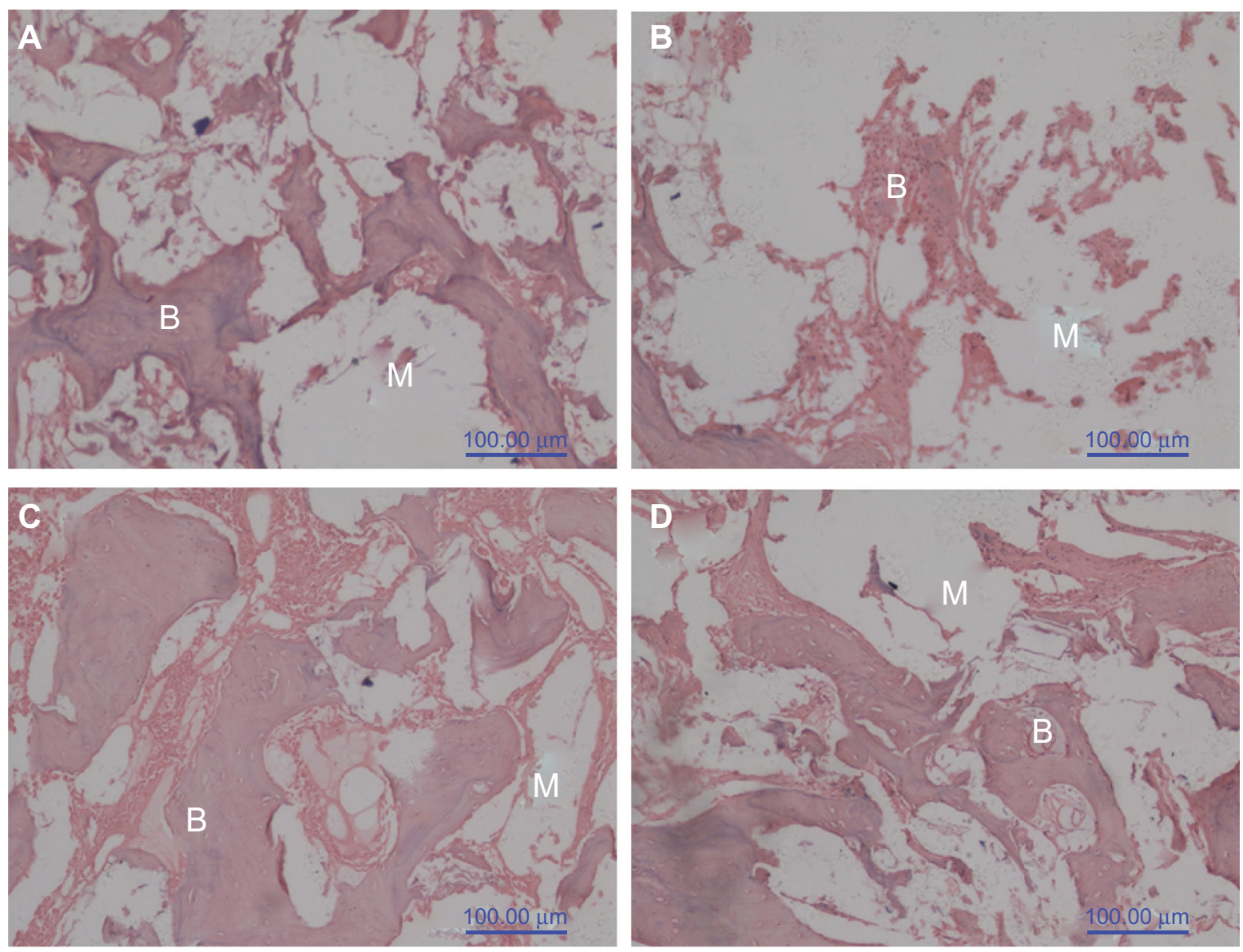

Figure 9 Hematoxylin and eosin stained section of (A, C) nanofluorapatite (n-FA)/polyamide I2 (PAI2) composite with 40 wt\% n-FA and (B, D) PAI2 implanted into bone defects of rabbit femora for (A, B) 4 weeks and (C, D) 8 weeks.

Note: $B$ represents new bone tissue and $M$ represents gradually degraded materials.

It was reported that ionic dissolution products containing fluoride from bioactive glasses can stimulate osteoblast proliferation. ${ }^{26}$ Moreover, a study has shown that HA substituted with fluorine had higher osteoblastic cellular activity, and micromolar concentrations of fluorine were effective to stimulate cell proliferation and differentiation. ${ }^{7,26}$ In this study, MTT test results showed that the n-FA/PA12 com-

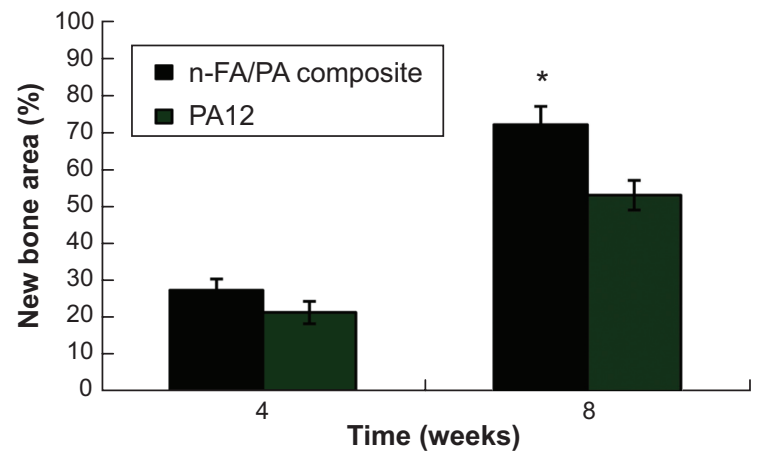

Figure 10 Percentage of newly formed bone area in both nanofluorapatite (n-FA)/ polyamide 12 (PAI2) composite with 40 wt $\%$ n-FA and PAI 2 at 4 weeks and 8 weeks. Note: *Indicates significant difference.

Abbreviation: PAI2, nanofluorapatite (n-FA)/polyamide 12 composite. posite obviously stimulated MC3T3-E1 cell growth and proliferation, and cell proliferation for n-FA/PA12 composite was significantly higher than for PA12. Regarding the exact concentration of fluorine that is appropriate for cell responses, there is still controversy over the range $10^{-5} \sim 10^{-7} \mathrm{M} \cdot{ }^{27,28} \mathrm{FA}$ is considered to release the optimal amount of fluorine for cellular activity, and the concentrations of fluorine released from FA were all within this range. ${ }^{29}$

For bone repair purposes, stimulating the osteogenic differentiation of mesenchymal stem cells is very important to the successful osseointegration for the implants with the surrounding bone tissue. ${ }^{30}$ Studies have shown that FA could support the initial adhesion and growth of MG63 cells and stimulate the differentiation of MG63 cells. ${ }^{31}$ In this study the ALP activity of the MC3T3-E1 cells on the n-FA/PA12 composite exhibited significantly higher expression levels than PA12, indicating that the cells differentiated more quickly on n-FA/PA12 composite than PA12. The results revealed that, compared with PA12, the improved ALP activity of the MC3T3-E1 cells on n-FA/PA12 composite was because of the presence of n-FA in 
the composite or slow release of fluoride ions from the n-FA that might be responsible for stimulating cell proliferation and differentiation. Therefore, it can be expected that n-FA/ PA12 composite might have the better function to promote cell/tissue responses than PA12 when implanted in vivo.

It has been found that pores between $50 \mu \mathrm{m}$ and $150 \mu \mathrm{m}$ determine osteoid growth, and pores around $500 \mu \mathrm{m}$ facilitate proliferation of cells, vascular ingrowth, and internal mineralized bone formation. In this study the porous scaffolds of n-FA/PA12 composite were fabricated by injection foaming method. These scaffolds exhibited a homogeneous distribution of open macropores, with pore sizes mainly in the range of $400-500 \mu \mathrm{m}$. Therefore, the characteristics of the scaffolds were beneficial in facilitating cell infiltration and bone ingrowth. In the present study the results confirmed that the MC3T3-E1 cells attached intimately and spread well on the n-FA/PA12 composite scaffold surfaces, forming confluent layers across the macroporous scaffolds. In-depth examination of the scaffolds revealed that these cells were clustered along the macroporous walls and penetrated into the composite scaffolds. This might allow cellular infiltration into the interior of the scaffolds and to increase the invasion of surrounding tissue when implanted in vivo.

However, only a few MC3T3-E1 cells attached and spread on the wall surface of composite scaffold. Therefore, the results demonstrated that n-FA/PA12 composite scaffold might have the better performance to promote cell attachment, spreading, and ingrowth as compared with PA12.

It is known that fluoride can increase bone density, and has effectiveness in prevention of fracture of human bone. ${ }^{32,33}$ Moreover, fluoride is a substance with significant biological activity that can stimulate bone formation. ${ }^{34,35}$ Therefore, fluoride-based biomedical implants might be of interest for bone repair. In this study the in vivo bioperformances of the n-FA/PA12 composite were investigated using the rabbit model (bone defect), and histological elevations were performed on the n-FA/PA12 composite scaffold after different implantation periods, in order to monitor the process of new bone tissue formation. The results showed that active bone regeneration was found in the bone defects of rabbits in this study, and new bone regenerated and gradually penetrated into the n-FA/PA12 composite scaffolds was observed as the implantation time prolonged, showing that the n-FA/PA1 2 composite enhanced the efficiency of bone formation. In addition, $28 \% \pm 3 \%$ and $62 \% \pm 5 \%$ of the bone defect area was found to be filled with newly formed bone tissue after n-FA/PA12 composite scaffolds implanted for 4 weeks and 8 weeks, respectively, indicating that n-FA/PA12 composite could promote osteogenesis in vivo. However, the area of newly formed bone was $21 \% \pm 3 \%$ and $53 \% \pm 4 \%$ after PA12 scaffold implanted for 4 weeks and 8 weeks, respectively. The results demonstrated that the n-FA/PA12 composite could stimulate bone regeneration and exhibit high efficiency of new bone formation. It can be suggested that the n-FA/PA12 composite presented not only good biocompatibility but also faster and more effective osteogenesis by incorporation of n-FA into PA12 as compared with PA12. This study opens up a new possibility for bone repair with n-FA-based composite in orthopedic surgery.

\section{Conclusion}

In this study the n-FA/PA12 composite was prepared by cosolution method. The results indicated that incorporation of n-FA into PA12 was an effective way to improve the mechanical properties (such as compressive strength and elastic modulus) and hydrophilicity of n-FA/PA12 composite, which were obviously higher than PA12. In addition, compared with PA12, the n-FA/PA12 composite had good antibacterial properties, which could meet the needs of antibacterial implanted materials, preventing bacterial infections. The proliferation and ALP activity of MC3T3-E1 cells on the n-FA/PA12 were significantly higher than for PA12, indicating that n-FA/PA12 composite could promote cell proliferation and differentiation. The histological evaluation results confirmed that n-FA/PA12 composite could stimulate new bone formation, and the quantity of the newly formed bone for n-FA/PA12 composite was significantly higher than for PA12, which exhibited high efficiency of bone regeneration in vivo. In short, n-FA/PA12 composite with good mechanical, hydrophilic, and antibacterial properties exhibited good biocompatibility and osteogenesis. It is expected that n-FA/PA12 composite as orthopedic implants and prostheses might be suitable for application in orthopedic surgery.

\section{Acknowledgments}

The authors appreciate financial support by the Hangzhou Science and Technology Bureau (No. 20110733Q30), the National Natural Science Foundation of China (Nos. 81271705, 83171383, and 51173041), the Key Medical Program of Science and Technology Development of Shanghai (No. 12nm0500400, 12441903600), and Key Projects of Basic Research of Science and Technology Development of Shanghai (No. 12JC1411302).

\section{Disclosure}

The authors report no conflicts of interest in this work. 


\section{References}

1. Swetha M, Sahithi K, Moorthi A, Srinivasan N, Ramasamy K, Selvamurugan N. Biocomposites containing natural polymers and hydroxyapatite for bone tissue engineering. Int J Biol Macromo. 2010;147:1-4.

2. Yang L, Perez-Amodio S, Barrere-de groot FY, Everts V, Blitterswijk van CA, Habibovic P. The effects of inorganic additives to calcium phosphate on in vitro behavior of osteoblasts and osteoclasts. Biomaterials. 2010;31:2976-2989.

3. Busch S, Schwarz U, Kniep R. Morphogenesis and structure of human teeth in relation to biomimetically grown fluorapatite-gelatine composites. Chem Mater. 2001;13:3260-3271.

4. Azami M, Jalilifiroozinehad S, Mozafari M, Rabiee M. Synthesis and solubility of calcium fluoride/hydroxy-fluorapatite nanocrystals for dental applications. Ceram Inter. 2011;237:2007-2014.

5. Wang HY, Sun KN, Shan T, Yang XQ, Zhao Y, Liang YJ. Biomimetic synthesis of fluorapatite coating. Adv Mater Res. 2011;306:63-71.

6. Brauer DS, Karpukhina N, O'Donnell MD, Law RV, Hill RG. Fluoridecontaining bioactive glasses: effect of glass design and structure on degradation, $\mathrm{pH}$ and apatite formation in simulated body fluid. Acta Biomater. 2010;6:3275-3282.

7. Cooper LF, Zhou Y, Takebe J, et al. Fluoride modification effects on osteoblast behavior and bone formation at $\mathrm{TiO}_{2}$ grit-blasted c.p. titanium endosseous implants. Biomaterials. 2006;27:926-936.

8. Khan A, Aamer S, Chaudhry A, Wong F, Rehman I. Synthesis and characterizations of a fluoride-releasing dental restorative material. Mater Sci Eng C Mater Biol Appl. 2013;33:3458-3464.

9. Takagi S, Frukhtbeyn S, Chow LC, Sugawara A, Fujikawa K, Ogiso BJ. In vitro and in vivo characteristics of fluorapatite-forming calcium phosphate cements. J Res Natl Inst Stand Technol. 2010;115: 267-276.

10. Puppi D, Chiellini F, Piras AM, Chiellini E. Polymeric materials for bone and cartilage repair. Prog Polym Sci. 2010;35:403-440.

11. Sahoo NG, Pan YZ, Li L, He CB. Nanocomposites for bone tissue regeneration. Nanomed. 2013;8:639-653.

12. Gerhardt LC, Widdows KL, Erol MM, et al. The pro-angiogenic properties of multi-functional bioactive glass composite scaffolds. Biomaterials. 2011;32:4096-4108.

13. Boccaccini AR, Erol M, Stark WJ, Mohn D, Hong Z, Manoe JF. Polymer/bioactive glass nanocomposites for biomedical applications: a review. Compos Sci Technol. 2010;270:1764-1776.

14. Colombo P, Mera G, Riedel R, Soraru GD. Polymer-derived ceramics: 40 years of research and innovation in advanced ceramics. J Am Ceram Soc. 2010;293:805-1837.

15. Li J, Sun H, Sun D, Yao Y, Yao F, Yao K. Biomimetic multicomponent polysaccharide/nano-hydroxyapatite composites for bone tissue engineering. Carbohydr Polym. 2011;85:885-894.

16. Dorozhkin SV. Bioceramics of calcium orthophosphates. Biomaterials. 2010;31:1465-1485.

17. Kushwaha M, Pan XL, Holloway JA, Denry IL. Differentiation of human mesenchymal stem cells on niobium-doped fluorapatite glassceramics. Dent Mater. 2012;28:252-260.

18. Chen Y, Miao X. Thermal and chemical stability of fluorohydroxyapatite ceramics with different fluorine contents. Biomaterials. 2005;26: $1205-1210$.
19. Qu Y, Wang P, Man Y, Li Y, Zuo Y, Li J. Preliminary biocompatible evaluation of nano-hydroxyapatite/polyamide 66 composite porous membrane. Int J Nanomedicine. 2010;5:429-435.

20. Huang D, Zuo Y, Li J, et al. Bioactive composite gradient coatings of nano-hydroxyapatite/polyamide 66 fabricated on polyamide 66 substrates. J R Soc Interface. 2012;9:1450-1457.

21. Kheradmanfard M, Fathi MH. Preparation and characterization of Mg-doped fluorapatite nanopowders by sol-gel method. J Alloys Compd. 2010;504:41-45.

22. Bhadang KA, Holding CA, Thissen H, Mclean KM, Forsythe JS, Haynes DR. Biological responses of human osteoblasts and osteoclasts to flame-sprayed coatings of hydroxyapatite and fluorapatite blends. Acta Biomater. 2010;6:1575-1583.

23. Simon P, Schwarz U, Kniep R. Hierarchical architecture and real structure in a biomimetic nano-composite of fluorapatite with gelatine: a model system for steps in dentino-and osteogenesis. J Mater Chem. 2005; 15:4992-4996.

24. Nordquist WD, Krutchkoff DJ. Part III: crystalline fluorapatite-coated hydroxyapatite; potential use as a bacteriostatic agent for both preimplant cases and retreatment of infected implant sites: a report of 4 cases. J Oral Implant. 2011;37:43-51.

25. Breaker RR. New insight on the response of bacteria to fluoride. Caries Res. 2012;46:78-81.

26. Kim FM, Hayes C, Williams PL, et al. An assessment of bone fluoride and osteosarcoma. J Dent Res. 2011;90:1171-1176.

27. Vrouwenvelder WCA, Groot CG, deGroot K. Better histology and biochemistry for osteoblasts cultured on titanium-doped bioactive glass: bioglass 45S5 compared with iron-, titanium-, fluorine-and boron-containing bioactive glasses. Biomaterials. 1994;15:97-106.

28. Ohno M, Kimoto K, Toyoda T, Kawata K, Arakawa H. Fluoride-treated bio-resorbable synthetic hydroxyapatite promotes proliferation and differentiation of human osteoblastic MG-63 cells. J Oral Implant. 2013:39:154-160

29. Li J, Song Y, Zhang S, et al. In vitro responses of human bone marrow stromal cells to a fluoridated hydroxyapatite coated biodegradable Mg-Zn alloy. Biomaterials. 2010;31:5782-5788.

30. Seong JM, Kim BC, Park JH, Kwon IK, Mantalaris A, Hwang YS. Stem cells in bone tissue engineering. Biomed Mater. 2010;5:062001.

31. Yoon BH, Kim HW, Lee SH, et al. Stability and cellular responses to fluorapatite-collagen composites. Biomaterials. 2005;26:2957-2963.

32. Liu J, Jin T, Chang S, et al. The effect of novel fluorapatite surfaces on osteoblast-like cell adhesion, growth, and mineralization. Tissue Eng. 2010;16:2977-2986.

33. Bhadang KA, Holding CA, Thissen H, McLean KM, Forsythe JS, Haynes DR. Biological responses of human osteoblasts and osteoclasts to flame-sprayed coatings of hydroxyapatite and fluorapatite blends. Acta Biomater. 2010;6:1575-1583.

34. Kansal I, Goel A, Tulyaganov DU, et al. Diopside $\left(\mathrm{CaO} \cdot \mathrm{MgO} \cdot 2 \mathrm{SiO}_{2}\right)-$ fluorapatite $\left(9 \mathrm{CaO} \cdot 3 \mathrm{P}_{2} \mathrm{O}_{5} \cdot \mathrm{CaF}_{2}\right)$ glass-ceramics: potential materials for bone tissue engineering. J Mater Chem. 2010;21:16247-16156.

35. Bandyopadhyay-Ghosh S, Reaney IM, Brook IM, Hurrell-Gillingham K, Johnson A, Hatton PV. In vitro biocompatibility of fluorcanasite glass-ceramics for bone tissue repair. J Biomed Mater Res. 2007;80: 175-183.
International Journal of Nanomedicine

\section{Publish your work in this journal}

The International Journal of Nanomedicine is an international, peerreviewed journal focusing on the application of nanotechnology in diagnostics, therapeutics, and drug delivery systems throughout the biomedical field. This journal is indexed on PubMed Central, MedLine, CAS, SciSearch ${ }^{\circledR}$, Current Contents ${ }^{\circledR} /$ Clinical Medicine,

\section{Dovepress}

Journal Citation Reports/Science Edition, EMBase, Scopus and the Elsevier Bibliographic databases. The manuscript management system is completely online and includes a very quick and fair peer-review system, which is all easy to use. Visit http://www.dovepress.com/ testimonials.php to read real quotes from published authors. 